\title{
ACRL Candidates, 1983 Elections
}

The listing for each of the following candidates includes the title, institution, and institutional address.

\section{Vice-President/President-Elect}

Keith M. Cottam, Associate Director, Vanderbilt University Library, Nashville, TN 37203; Sharon J. Rogers, Director of Public Services, University Libraries, Bowling Green State University, Bowling Green, OH 43403.

\section{Anthropology and Sociology Section (ANSS)}

Vice-Chair/Chair-elect: Bonnie R. Nelson, Assistant Professor, Library, John Jay College of Criminal Justice, New York, NY 10012; Lynne M. Schmelz-Keil, Reference and Audiovisual Librarian, John Cotton Dana Library, Rutgers University, Newark, NJ 07102.

Secretary (one-year term): Christopher J. Busick, Reference Librarian, Anthropology and Economics Bibliographer, Norlin Library, University of Colorado, Boulder, CO 80309; Marvin D. Leavy, Behavioral Sciences Librarian, Wichita State University Library, Wichita, KS 67208.

Member-at-Large (two-year term): Hazel Johnson, Social Sciences Bibliographer, Hillman Library, University of Pittsburgh, Pittsburgh, PA 15260; Linda Katzoff-Grodofsky, Reference/Collection Development Librarian for Anthropology and Sociology, Samuel Paley Library, Temple University, Philadelphia, PA 19122.

\section{Art Section}

Vice-Chair/Chair-elect: GraceAnne A. DeCandido, Coordinator, Archon Project, Technical Service Division, Bobst Library, New York University, New York, NY 10012.

Secretary (two-year term): D. Sherman Clarke, Head, Humanities Cataloging Team, Olin Library, Cornell University Libraries, Ithaca, NY 14853; Lisa Howorth, Reference-Bibliographer and Instructor, J. D. Williams Library, University of Mississippi, University, MS 38677.

\section{Asian and African Section (AAS)}

Vice-Chair/Chair-elect: Basima Q. Bezirgan, Middle East Cataloger, Joseph Regenstein Library, University of Chicago, Chicago, IL 60637; Martha Dukas, Coordinator of Processing, Boston Public Library, Boston, MA 02117.

Member-at-Large (three-year term): Gia Aivazian, Librarian for Armenian and Greek, Technical Services Department, Research Library, University of California, Los Angeles, CA 90024; John Yung-hsiang Lai, Associate Librarian, HarvardYenching Library, Harvard University, Cambridge, MA 02138; Joanne M. Zellers, Area Spe- cialist, African and Middle Eastern Division, Africa Section, Library of Congress, Washington, DC 20540.

\section{Bibliographic Instruction Section (BIS)}

Vice-Chair/Chair-elect: Beverly L. Renford, Reference Librarian, Hershey Medical Center Library, Pennsylvania State University, Hershey, PA 17033; William Miller, Head of Reference, Michigan State University Library, East Lansing, MI 48224-1048.

Secretary: Patricia L. Bril, Reference Librarian/Assistant to the University Librarian, California State University Library, Fullerton, CA 92634; Roland C. Person, Assistant Undergraduate Librarian, Southern Illinois University, Carbondale, IL 62901.

Member-at-Large (one-year term): Claudette S. Hagle, Assistant Professor/Assistant Reference Librarian, Oklahoma State University Library, Stillwater, OK 74078; Barbara J. Wittkopf, Associate Librarian, Department of Reference and Bibliography, University of Florida Libraries, Gainesville, FL 32611.

\section{College Library Section (CLS)}

Vice-Chair/Chair-elect: Michael Haeuser, Director of Learning Resources, Gustavus Adolphus College, St. Peter, MN 56082; William A. Moffett, Director of Libraries, Oberlin College, Oberlin, OH 44074.

Secretary (one-year term): Larry J. Frye, Head Librarian, Lilly Library, Wabash College, Crawfordsville, IN 47933; Vicki R. Kreimeyer, Assistant Director for Public Services, Lewis and Clark College Library, Portland, OR 97219.

College and Junior College Library Section (CJCLS)

Vice-Chair/Chair-elect: Elinor Ebeling, Dean, Learning Resources, Brookdale Community College, Lincroft, NJ 07738; Iole Matteucig, Dean, Library Services, City College of San Francisco, San Francisco, CA 94112.

Secretary (one-year term): Robert B. Ford, Chief Librarian, Medgar Evers College Library, Brooklyn, NY 11225; Mildred D. Kirsner, Associate Professor, Reference Librarian-Coordinator, Library Instruction, Miami Dade Community College, North Campus Library, Miami, FL 33167 .

Education and Behavioral Sciences Section (EBSS)

Vice-Chair/Chair-elect: L. James Olivetti, Library Systems Analyst, Evaluation Technologies, Inc., Arlington, VA 22201; Thomas M. Peischl, 
Director of College Libraries and Computing Services, State University of New York College at Potsdam, Potsdam, NY 13676.

Secretary (two-year term): Annette Buurstra, Education Librarian, Northeastern Illinois University Library, Chicago, IL 60625; Ilene R.'Rockman, Reference Librarian, Learning Resources and Curriculum Department, California Polytechnic State University, San Luis Obispo, CA 93407.

\section{Law and Political Science Section (LPSS)}

Vice-Chair/Chair-elect: Peter Malanchuk, Chairman, Department of Reference and Bibliography, University Libraries, University of Florida, Gainesville, FL 32611; Laurene E. Zaporozhetz, Assistant Professor/Reference Librarian, University of Oregon Library, Eugene, OR 97403.

Member-at-Large: Marva L. DeLoach, Technical Services Librarian, Co-Director of the Social Science Student Scholars Project and Associate Professor, Huntington Library, Hampton Institute, Hampton, VA 23668; Ray L. Morrison, Bibliographic Instruction Librarian, Pittsburg State University, Pittsburg, KS 66762.

\section{Rare Books and Manuscripts Section (RBMS)}

Vice-Chair/Chair-elect: Lynda Corey Claassen, Coordinator, The Center for the Book, The Library of Congress, Washington, DC 20540; David Farmer, Director, Rare Books and Special Collections, McFarlin Library, University of Tulsa, Tulsa, OK 74104.

Secretary (two-year term): Anthony Bliss, Rare
Book Librarian, Bancroft Library, University of California, Berkeley, CA 94720; David S. Zeidberg, Curator of Special Collections, Gelman Library, George Washington University, Washington, DC 20052.

Member-at-Large (three-year term): C. Daniel Elliott, Assistant Head, Department of Special Collections, University of California Library, Davis, CA 95616; Jennifer B. Lee, Reference Librarian, Library Company of Philadelphia, Philadelphia, PA 19107.

\section{Science and Technology Section (STS)}

Vice-Chair/Chair-elect: Linda L. Phillips, Acting Head, Undergraduate Library, The University of Tennessee, Knoxville, TN 37996; Keith E. Roe, Head, Life Sciences Library, The Pennsylvania State University, University, PA 16802.

Secretary (one-year term): Sheila Grant Johnson, Head, Biological Science Division, Oklahoma State University Library, Stillwater, OK 74078; Martin Kesselman, Life Sciences Librarian, Coles Science Center-Bobst Library, New York University, New York, NY 10012.

\section{Slavic and East European Section (SEES)}

Vice-Chair/Chair-elect: Ivan L. Kaldor, Professor, School of Library and Information Science, State University College of Arts and Science, Geneseo, NY 14454; Laszlo Kovacs, Head, Humanities Library, Purdue University, West Lafayette, IN 47907.

Member-at-Large (one-year term): Donna A. Canevari, Slavic Bibliographer, Collection Devel-

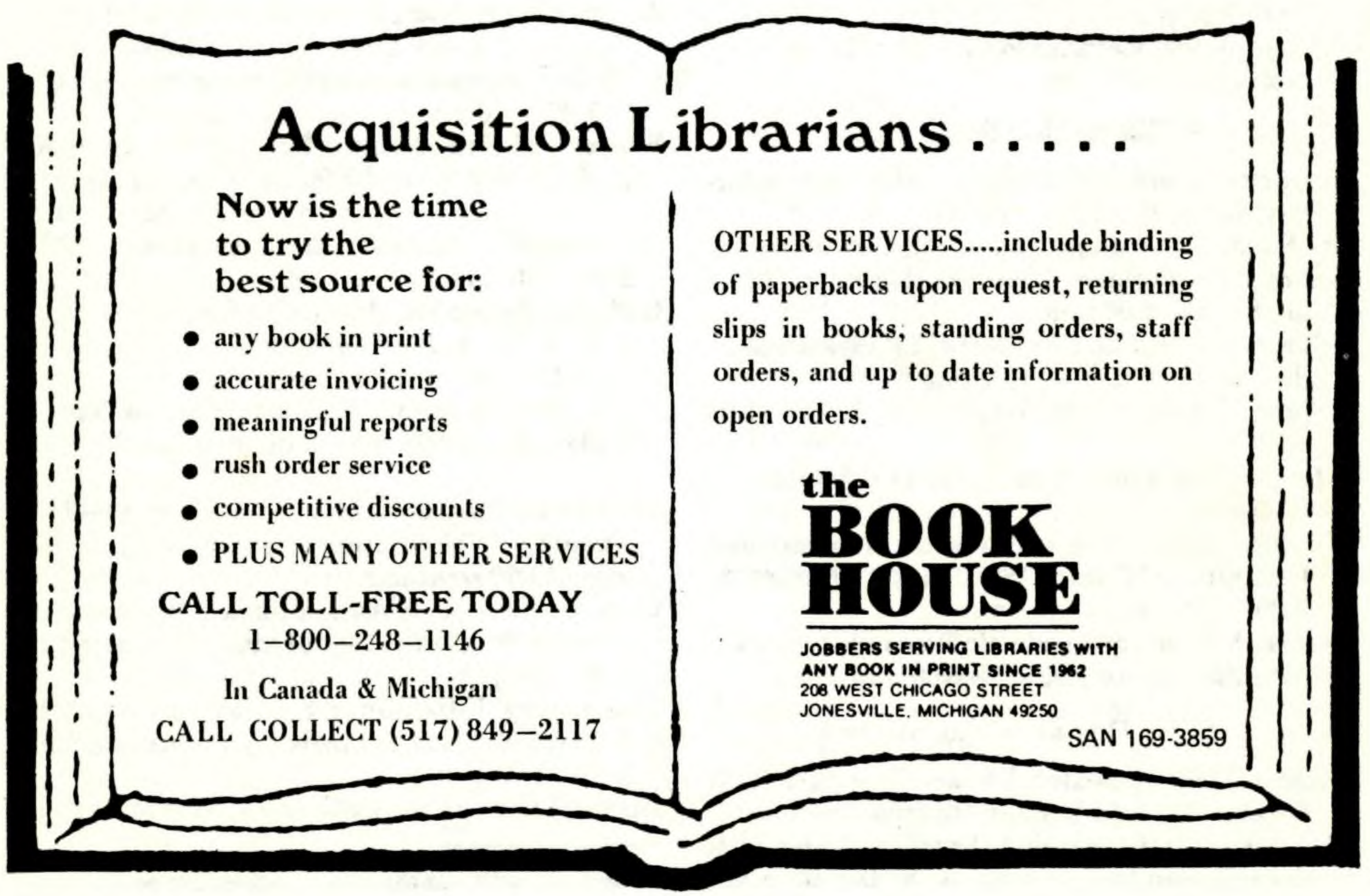


opment, Main Library, University of Saskatchewan, Saskatoon, Saskatchewan, S7N 0W0, Canada; Dennis Kimmage, Senior Assistant Librarian, Feinberg Library, State University College, Plattsburgh, NY 12901.

\section{UNIVERSITy LibraRIES SECTION (ULS)}

Vice-Chair/Chair-elect: Cynthia B. Duncan, Dean of Library Services, Old Dominion University, Norfolk, VA 23508; Melvin R. George, University Librarian and Director Learning Services, Northeastern Illinois University, Chicago, IL 60625.

Secretary (three-year term): Janice Koyama, Head Librarian, Moffitt Undergraduate Library, University of California, Berkeley, CA 94720; Ann K. Randall, Assistant University Librarian, Brown University, Providence, RI 02912.
Western European Specialists Section (WESS)

Vice-Chair/Chair-elect: Ross Atkinson, $\mathrm{Hu}-$ manities Bibliographer, Collection Management Division, Northwestern University Library, Evanston, IL 60201; Cecily Johns, Director of Collections and Information Services, University of Cincinnati, Cincinnati, $\mathrm{OH} 45221$.

Secretary (one-year term): Jeffry Larson, $\mathrm{Hu}$ manities Bibliographer, Yale University Library, New Haven, CT 06520; Fred J. Gitner, Librarian, French Institute/Alliance Francaise Library, New York, NY 10022.

Member-at-Large (one-year term): Marjorie A. Benedict, Bibliographer, State University of New York at Albany, Albany, NY 12222; Laszlo Kovacs, Head, Humanities Library, Purdue University, West Lafayette, IN 47907.

\section{Tentative Schedule of}

ACRL Midwinter Meetings

ALA Midwinter Meeting

San Antonio, Texas

JANUARY 8-13, 1983

\section{ACRL BOARD OF DiRECTORS}

First Board Meeting: Sunday, January 9, 2:00-5:30 p.m.

Second Board Meeting: Wednesday, January 12, 9:30 a.m. $-12: 30$ p.m

\section{ACRL Ad Hoc Task Forces}

Academic Libraries and Higher Education: Saturday, January 8, 2:00-4:00 p.m.

ACRL/ALA: Saturday, January 8, 2:00-4:00 p.m.

Library Performance Measures: Saturday, January 8, 2:00-4:00 p.m.

Library Schools and Academic Libraries: Saturday, January 8, 2:00-4:00 p.m.

Library Statistics: Saturday, January 8, 2:00-4:00 p.m.

Membership Promotion: Saturday, January 8, 8:00 a.m. $-12: 30$ p.m.

Orientation of New Committee Members and Committee Chairs: Saturday, January 8, 2:00-4:00 p.m.

Research Needs of Academic/Research Libraries: Saturday, January 8, 2:00-4:00 p.m.

\section{ACRL Divisional Committees}

Academic and Research Library Personnel Study Group: Monday, January 10, 8:00-11:00 a.m.

Academic or Research Librarian of the Year Award: Sunday, January 9, 8:00-9:00 a.m.;
Monday, January 10, 9:30 a.m.-12:30 p.m. *

Academic Status: Saturday, January 8, 8:00-10:00 p.m.; Wednesday, January 12, 8:00-11:00 a.m.

Appointments and Nominations: Saturday, January 8, 2:00-4:00 p.m.; Sunday, January 9, 9:30-11:00 a.m.*

Audiovisual: Sunday, January 9, 9:30-11:00 a.m.; Tuesday, January 11, 11:30 a.m.-12:30 p.m.

Books for College Libraries III: Saturday, January $8,5: 30-7: 00$ p.m.

Budget and Finance: Monday, January 10, 9:30 a.m.-12:30 p.m. and 2:00-5:30 p.m.; Tuesday, January 11, 9:30 a.m.-12:30 p.m. and 2:00-5:30 p.m.; Wednesday, January 12, 2:00-5:30 p.m.

College Library Standards: Sunday, January 9, 2:00-4:00 p.m.; Monday, January 10, 9:00-11:00 a.m.

Conference Program Planning CommitteeDallas, 1984: Monday, January 10, 8:00-9:00 a.m.

Conference Program Planning Committee-Los Angeles, 1983: Sunday, January 9, 11:30 a.m. $-12: 30$ p.m.

Constitution and Bylaws: Monday, January 10, 9:30-11:00 a.m.; Wednesday, January 12, 2:00-4:00 p.m.

Continuing Education: Sunday, January 9, 9:00-11:00 a.m.; Monday, January 10, 9:00-11:00 a.m.

Doctoral Dissertation Fellowship: Monday, Janu-

*Meetings with an asterisk are closed meetings. 